\title{
Comparison of two fast neutron fluence measurement methods based on Np-237 fission-to-capture ratio measurement (spectral index) and a reverse dark current measurement in a planar sili- con detector
}

\author{
Marcin Szuta ${ }^{1, \star}$, Stanislaw Kilim¹ , Elzbieta Strugalska-Gola1 ${ }^{1}$, Marcin Bielewicz ${ }^{1,2}$, Niko- \\ lay Zamyatin ${ }^{2}$, Anastasia Shafronovskaia ${ }^{2}$, and Sergey Tyutyunnikov ${ }^{2}$ \\ ${ }^{1}$ National Centre for Nuclear Research, 05-400 Otwock-Świerk, Poland \\ ${ }^{2}$ Joint Institute for Nuclear Research, 141980 Dubna, Russia
}

\begin{abstract}
The idea of the first method is to search the neutron energy for the ratio of fission cross section to capture cross section of the selected actinide isotope from the nuclear data base that is equal to the measured ratio of the fissioned and captured actinide isotope $\mathrm{Np}-237$.

The idea of the second method consists in the measurement of the reverse dark current increase, which is linearly proportional to neutron fluence, induced by the fast neutron irradiation in planar silicon detectors.

$\mathrm{Np}$-237 samples and planar silicon detectors were placed inside a subcritical assembly (the Quinta assembly at the Joint Institute for Nuclear Research, Russia) very close to each other assuming that in both samples the same neutron fluence should pass.

We concluded that minor actinide samples and planar silicon detectors can be used as neutron fluence detectors especially in the high neutron energy range, where measurements are difficult.

Considering the importance of high energy neutron measurement in the ADS (Accelerator Driven System), actinide and silicon detectors could be a very useful tool.
\end{abstract}

\section{Introduction}

Minor actinides (MA) (Np, Am, Cm) are generated in nuclear fuel during irradiation in a reactor. These elements significantly contribute in radio-toxicity and heat generation of spent nuclear fuel. The key issue for reducing the MA radio-toxicity is the partitioning and transmutation of MA in fast neutron reactors (FR) or accelerator driven systems (ADS).

The problem is to determine the optimal place in the ADS for incineration of actinides. This can be done by application of actinides and silicon semiconductors as detectors of fast neutron fluence.

The Quinta experimental assembly simulating a deeply sub-critical assembly, located at the Joint Institute for Nuclear Research (JINR), Dubna, Russia, was used for preliminary application of the fast neutron spectrum to the actinides and silicon semiconductors to study the neutron fluence. The Quinta target was irradiated with a pulsed proton extracted from the Phasotron accelerator.

^e-mail: marcin.szuta@ncbj.gov.pl 


\section{Fast neutron fluence measurement methods}

\subsection{Actinide fast neutron fluence measurement method}

Since the measured spectral indexes are defined as the ratio of average fission and capture cross sections, the retrieved distinct fission and capture cross sections for the distinct neutron energy from the nuclear data base describe the average values.

Having the average fission and capture cross section values, we can evaluate the average neutron flux in the location of the actinide sample using the measured amount of fissioned and captured actinide isotopes. Two different equations for fissioned $\left(N_{y f}\right)$ and captured $\left(N_{y c}\right)$ actinide isotopes should give the same average neutron flux value, that is a proof for correct measurement.

The number of neutron induced fissioned $\left(N_{y f}\right)$ and neutron captured actinide isotopes $\left(N_{y c}\right)$ in the actinide sample of volume $V_{p}$ can be expressed as follows:

$$
N_{y f}=V_{p} \bar{\Phi} N \bar{\sigma}_{f} t \quad ; \quad N_{y c}=V_{p} \bar{\Phi} N \bar{\sigma}_{c} t
$$

where

$\bar{\Phi}$ - average neutron flux in the place of the actinide sample location $\left[\mathrm{n} / \mathrm{cm}^{2} \cdot s\right]$,

$N$ - number of actinide isotopes in a volume unit $\left[\mathrm{cm}^{-3}\right]$,

$\overline{\sigma_{f}}, \overline{\sigma_{c}}$ - average microscopic cross section for the reactions $(n, f)$ or $(n, \gamma)$ [barns],

$t$ - irradiation time.

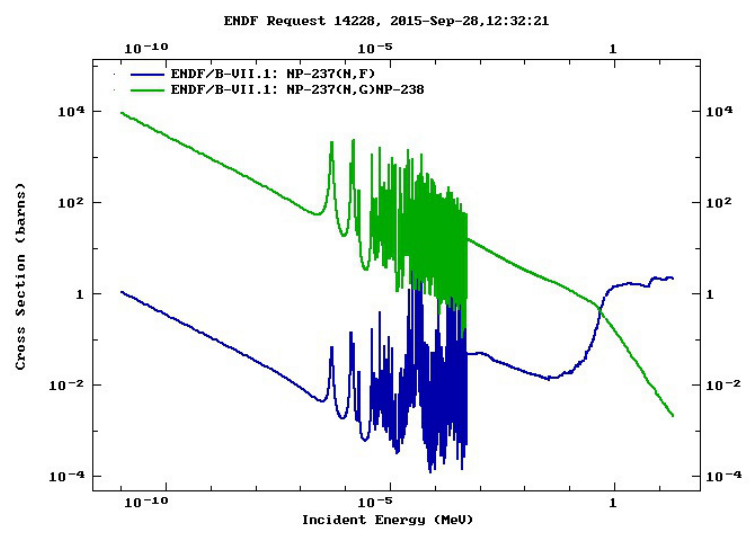

Figure 1. Cross sections of Np-237(n,g)Np-238 and Np-237(n,f) reactions.

The fission/absorption ratios are consistently higher for the fast spectrum. Thus, in a fast spectrum, actinides are preferentially fissioned, not transmuted into higher actinides (see figure 1).

\subsection{Silicon semiconductor fast neutron fluence measurement method}

Fast neutrons with an energy of $e n>100 \mathrm{keV}$ produce damage in the volume of the silicon semiconductor in the form of vacancies in the crystal lattice (knocking-out the primary atom from the lattice site).

The silicon detector changes its electrical parameters (characteristics) under neutron irradiation due to radiation defects in the crystal lattice. The radiation defects lead, among others, to reverse current growth.

The reverse dark current of the detector grows linearly [1] with increasing fast neutron fluence:

$$
\Delta I=\alpha_{I} \times V \times \Phi
$$


where:

$\Delta I=\left(I_{\text {irrad }}-I_{\text {nonirrad }}\right),[A]$, increment detector current,

$V$ - volume of the detector, $\left[\mathrm{cm}^{-3}\right]$,

$\Phi$ - neutron fluence, $\left[\mathrm{cm}^{-2}\right]$, - equivalent for silicon damage fluence of fast neutrons with an energy of $1 \mathrm{MeV}$,

$\alpha_{I}=(5 \pm 10 \%) 10^{-17}\left[\mathrm{~A} \mathrm{~cm}^{-1}\right]$, constant of radiation.

Neutron radiation induced damage in silicon detectors is presented in figure 2 . The values of neutron silicon detector damage cross section as a function of neutron energy presented in figure 2 are extracted from the figure described "Function of defect formation of $\mathrm{D}(\mathrm{E})$ in $\mathrm{MeV} \times \mathrm{mb}$,

(Ougouag) and the function NIEL-FN-522 in $\mathrm{keV} \times \mathrm{cm}^{2} / \mathrm{g}$ ", presented in reference [2].

The damage cross section in function of energy starts with the threshold mode at the energy of $170 \mathrm{keV}[2]$.

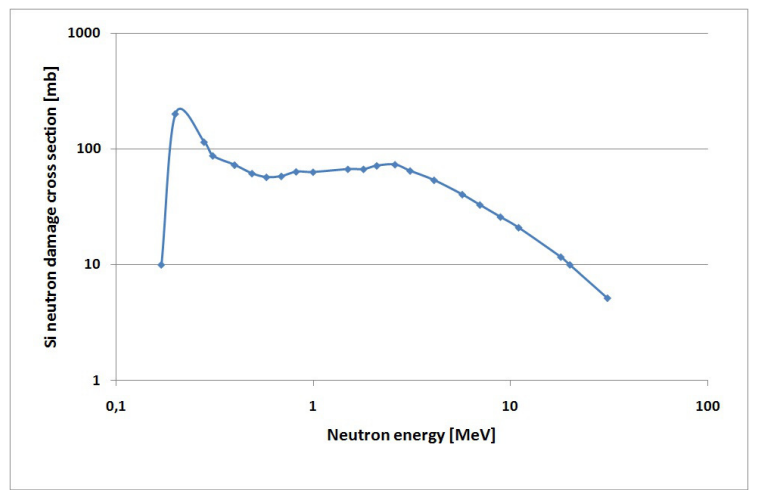

Figure 2. Neutron silicon detector damage cross section as a function of neutron energy [2].

We can roughly assume that in the neutron energy range from $0.4 \mathrm{MeV}$ to $4 \mathrm{MeV}$ the silicon detector damage cross section is constant which indicates that in this neutron energy range the precision of measured neutron fluence is defined by the changes of the damage cross section in the range, which is not greater than $25 \%$. This in turn implies that the calibration of the silicon detector with a neutron flux of any energy from this range is fulfilled within accuracy of $25 \%$. That is why the measured neutron fluence can be termed as an equivalent for silicon detector damage by fast neutrons with an energy of $1 \mathrm{MeV}$.

\section{Motivation of comparison of the two fast neutron fluence measurement methods}

Performing an experimental study of transmutation of actinides in a deeply sub-critical assembly controlled by an external neutron source (ADS) - the Quinta assembly, we proposed minor actinide application as a neutron fluence and average neutron energy detector in the place of their location in order to determine the optimal place in the ADS for the incineration of the actinides [3].

Independently, within the project "Study of deep subcritical electronuclear systems and feasibility of their application for energy production and radioactive waste transmutation - E\&T - RAW", a special JINR group concentrated on measuring fast neutron fluence using planar silicon detectors.

Accidentally, since location of some silicon detectors was very close to the location of the actinide Np-237 samples during certain experimental session and their results were quite comparable, we undertook to continue experimentally the comparison. 
The relationship between the neutron fluences measured for three different energies of deuteron beams ( 2,4 and $8 \mathrm{GeV}$ ) by the actinide detector and by the silicon detector is very alike. The values of the neutron fluencies measured by the actinide detectors are about 2.5 times higher than that measured by the silicon detectors. This is understandable because the silicon detectors measured the same neutron field, as the actinide detector, but weakened by $10 \mathrm{~cm}$ of a lead reflector. This observation motivated us for the comparison of the two fast neutron fluence measurement methods.

\section{Experimental study on (sounds strange, as comparison is a way of studying) the comparison of the two fast neutron fluence measurement methods}

\subsection{The Quinta assembly}

The Quinta assembly, located at the Joint Institute for Nuclear Research (JINR), Dubna, Russia, was used in the study of the actinide incineration for application of a fast neutron spectrum. It is an assembly of a massive uranium target and lead shielding (see figure 3). The Quinta assembly consists of a total of $512 \mathrm{~kg}$ of natural uranium. It is composed of five sections, each being $114 \mathrm{~mm}$ long and separated by a $17 \mathrm{~mm}$ air gap, which allows the placement of samples mounted onto special plates. The uranium exists as many cylindrical rods, where each rod is $36 \mathrm{~mm}$ in diameter, $104 \mathrm{~mm}$ in length and $1.72 \mathrm{~kg}$ in mass. The five sections are surrounded by $100 \mathrm{~mm}$ thick lead bricks on all six sides of a total weight of $1780 \mathrm{~kg}$. One of the sides of the lead shield-reflector has a rectangular window for placing the actinide samples (see figure 3 ) $[4,5]$.
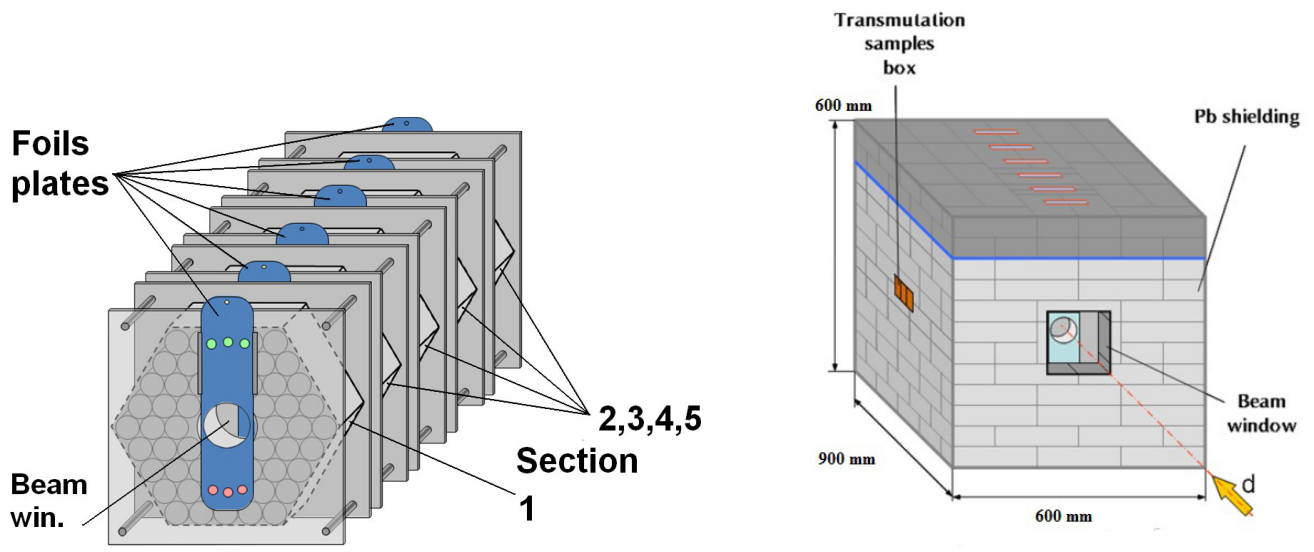

Figure 3. Scheme of the Quinta assembly. On the left there is a view of the uranium target with supporting structures and plastics used for sample placement (detector's plates), on the right there is a view of the lead shielding enfolding the target with transmutation samples box (left window) for the actinide sample placing in the shielding.

\subsection{Principles of the measurements}

After irradiation, the actinide samples were removed and transported away to be analyzed with gamma spectrometry. The actinide samples were measured using an HPGe n-type coaxial detector manufactured by CANBERRA. The measurements began $1.5 \mathrm{~h}$ after irradiation had stopped, continuing for up to 6 days afterwards. The spectra collection times ranged from $15 \mathrm{~min}$ to just over $3 \mathrm{~h}$. All spectra 
were analyzed using the DEIMOS [6] program. Spectroscopic corrections make about $1 \%$ uncertainty. Statistical uncertainty of the Gauss fit of the gamma peaks in the DEIMOS code depends on intensity and its value is usually between $1 \%$ and $10 \%$. Final uncertainty is set as a square root of the second powers of particular statistical and systematic uncertainties.

The total yield of the observed isotopes per one gram of activated material and one deuteron was calculated $N_{\text {yield }}$.

The procedure of evaluation of the actinide fission rate per deuteron and per gram $\left(I_{f \gamma}\right)$ consists in the calculation of the number of the produced nuclei $N_{\text {yield }}$ for each isotope and then dividing it by the isotope production efficiency per 1 fission $\left(\gamma_{f}\right)$ :

$$
I_{f \gamma}=N_{\text {yield }} / \gamma_{f}
$$

where

$I_{f \gamma}$ - actinide fission rate per deuteron and per gram.

The gamma-ray spectrum analysis was carried out in a well established manner. The measured activities of the considered isotopes at the end of irradiation (EOI) were corrected for the decay during the irradiation according to a known deuteron beam profile, for the decay between EOI and start of counting (cooling time) and for the decay during the counting time and also not forgetting about dead time correction $\left(t_{\text {real }} / t_{\text {live }}\right)$. Thus, one can calculate the relative number of the considered produced isotopes at the end of irradiation for each measurement.

\subsection{Irradiation details}

In order to compare the two methods, we performed an experiment where the silicon detector was placed very close to the ${ }^{237} \mathrm{~Np}$ sample in the rectangular window in the lead shielding (see figure 3 ) of the QUINTA assembly assuming that both detectors were irradiated by the same neutron flux.

The Quinta target was irradiated with a pulsed proton beam of energies of $0.66 \mathrm{GeV}$ extracted from the Phasotron accelerator. The total number of protons is equal to $8.64(30) \times 10^{14}$ during the time of irradiation equal to $20580 \mathrm{~s}$.

For the Np-237 sample of $1.147 \mathrm{~g}$ weight, the number of Np-237 fissions per sample $\left(N_{y f}\right)$ was equal to $1.458 \times 10^{10}$ and the number of captures per sample $\left(N_{y c}\right)$ was equal to $2.096 \times 10^{10}$. The measured ratio of fission to capture for the proton beam of energy of $0.66 \mathrm{GeV}$ is equal to $0.695 \pm 0.195$.

Applying the try-and-error method, we find the neutron energy for which the ratio of fission cross section to capture cross section of the actinide Np-237 from the nuclear data base (ENDF/B-VII.1) is equal to the measured ratio of the fissioned and captured actinide isotope. The retrieved distinct fission and capture cross sections for the distinct neutron energy from the nuclear data base, which describe the average values, let us evaluate the neutron fluencies using the equations (1).

Adding the values of neutron fluencies measured by the silicon detector method we can compare the two methods (see table 1).

Table 1. Compilation of neutron fluencies measured by the two methods

\begin{tabular}{|l|l|}
\hline Deuteron beam energy/year & $0.66 \mathrm{GeV} / 2014$ \\
\hline $\begin{array}{l}\text { Neutron fluence using for evaluation the number of fissions } \\
\text { in the sample }\left[\mathrm{n} / \mathrm{cm}^{2}\right] \times 10^{13}\end{array}$ & 1.67 \\
\hline $\begin{array}{l}\text { Neutron fluence using for evaluation the number of captures } \\
\text { in the sample }\left[\mathrm{n} / \mathrm{cm}^{2}\right] \times 10^{13}\end{array}$ & 1.64 \\
\hline Neutron fluence using silicon detectors $\left[\mathrm{n} / \mathrm{cm}^{2}\right] \times 10^{13}$ & 1.11 \\
\hline
\end{tabular}




\section{Discussion}

The mathematical structures of the equations describing the neutron fluence of the two methods are very alike. In both methods the values which are proportional to the neutron fluence depend on the cross section of the neutron inducing damage in the silicon detector and cross sections of the neutron inducing fission and capture on the Np-237 minor actinide (see figure 1 and figure 2). The cross sections used in both methods depending on the neutron energy are the primary reasons of the final result ( $I$ and $N_{y f}$ or $N_{y c}$ ) which let us evaluate the searched neutron fluence. The further similarity is that the volumes of the detectors in both methods are taken into account.

In the research, the fluence was estimated to be about $1013 \mathrm{n} / \mathrm{cm}^{2}$. The fluence measured by the silicon detector method was about $35 \%$ less than the fluence estimated by the actinide method. This could be expected because the silicon detector method allows effective measuring of the fast neutrons of an energy higher than $170 \mathrm{keV}$. So, the difference between the two fast neutron fluence measurements gives estimation of the neutron fluence for a neutron energy below $170 \mathrm{keV}$. This is an additional approach.

\section{Conclusion}

Minor actinide samples and planar silicon detectors can be used as neutron fluence detectors especially in the high neutron energy range where the measurements are impeded.

Given the importance of high-energy neutron measurements in the ADS (Accelerator Driven System), actinide and silicon detectors could be a very useful tool.

The difference between the two fast neutron fluence measurements of the two methods presented above gives, in addition, an estimation of the neutron fluence at neutron energy below $170 \mathrm{keV}$ - the additional approach.

\section{References}

[1] I.A. Golutvin et al., preprint E14-95-97, Dubna (1995)

[2] A. Van Ginneken, Non ionizing energy deposition in silicon for radiation damage studies (Fermi Nat. Accelerator Lab. Report FN-522, 1989)

[3] M. Szuta, S. Kilim, E. Strugalska-Gola, M. Bielewicz, S. Tyutyunnikov, Feasibility study of minor actinide application as a neutron fluency and average neutron energy detector in the place of their location, Fifth Meeting of the Expert Group on Reactor Physics and Advanced Nuclear System (EGRPANS) of Working Party on Scientific Issues of Reactor Systems (WPRS) (NEA Headquarters, Paris, France, 19 February 2015)

[4] "E\&T RAW" Collab. (I. Adam et al.) E1-2010-61, Dubna (2010)

[5] N.L. Asquith et al. Annals of Nuclear Energy 63, 742-750 (2014)

[6] J. Frana Radioanal. and Nucl. Chem. 257, 583 (2003) 